\title{
Trojan Horse particle invariance in fusion reactions
}

\author{
R.G. Pizzone ${ }^{1, a}$, C. Spitaleri ${ }^{12}$, C. Bertulani ${ }^{3}$, A. Mukhamedzhanov ${ }^{4}$, L. Blokhintsev ${ }^{5}$, M.La Cognata $^{13}$, L. Lamia ${ }^{13}$, \\ R. Spartá ${ }^{13}$, and A. Tumino ${ }^{1,6}$ \\ ${ }^{1}$ INFN-LNS, Catania, Italy \\ ${ }^{2}$ Dipartimento di Fisica e Astronomia, Universitá di Catania, Italy, \\ ${ }^{3}$ Department of Physics and Astronomy, Texas A\&M University-Commerce, Commerce, USA \\ ${ }^{4}$ Cyclotron Institute, Texas A\&M University, College Station,USA \\ ${ }^{5}$ Institute of Nuclear Physics, Moscow State University, Moscow, Russia \\ ${ }^{6}$ Università degli Studi di Enna "Kore", Enna, Italy
}

\begin{abstract}
Trojan Horse method plays an important part for the measurement of several charged particle induced reactions cross sections of astrophysical interest. In order to better understand its cornerstones and the related applications to different astrophysical scenarios several tests were performed to verify all its properties and the possible future perspectives. The Trojan Horse nucleus invariance for the binary reactions $\mathrm{d}(\mathrm{d}, \mathrm{p}) \mathrm{t}$, ${ }^{6,7} \mathrm{Li}(\mathrm{p}, \alpha){ }^{3,4} \mathrm{He}$ was therefore tested using the appropriate quasi free break- ups, respectively. In the first cases results from ${ }^{6} \mathrm{Li}$ and ${ }^{3} \mathrm{He}$ break up were used, while for the lithium fusion reactions break-ups of ${ }^{2} \mathrm{H}$ and ${ }^{3} \mathrm{He}$ were compared. The astrophysical S(E)-factors for the different processes were then extracted in the framework of the Plane Wave Approximation applied to the different break-up schemes. The obtained results are compared with direct data as well as with previous indirect investigations. The very good agreement between data coming from different break-up schemes confirms the applicability of the plane wave approximation and suggests the independence of binary indirect cross section on the chosen Trojan Horse nucleus also for the present cases. Moreover the astrophysical implications of the results will also be discussed in details.
\end{abstract}

\section{Introduction}

Fusion reactions induced by charged particles at astrophysical energies have many experimental difficulties, mainly connected to the presence of the Coulomb barrier and the electron screening effect. So several indirect methods have been developed, mainly based on direct reactions mechanisms (e.g. ANC [1, 2]) . Among them, an important role is played by the Trojan Horse Method (THM), usually applied at the energies of astrophysical interest, which is discussed extensively elsewhere [3-12, 12-18]. THM allows one to extract the low energy behavior of a binary reaction by applying the well known theoretical formalism of the Quasi-Free (QF) process, in the simplest cases. The basic idea of the THM is to extract the cross section in the low-energy region of a two-body reaction with significant astrophysical impact:

$$
A+x \rightarrow c+C
$$

from a suitable QF break-up of the so called Trojan Horse nucleus, e.g. $a=x \oplus s$ where usually $x$ is referred to as the participant and $s$ as the spectator particle. We refer to previous papers and references therein for an extensive discussion on THM and its theoretical formalism [20].

Many tests have been made to fully explore the potentiality of the method and extend as much as possible

\footnotetext{
ae-mail: rgpizzone@lns.infn.i
}

Table 1. Physical cases for which the Trojan Horse particle invariance was investigated. The relevant reference for each reaction is reported in the last column.

\begin{tabular}{llc}
\hline Quasi-free process & Trojan Horse particle & ref. \\
${ }^{6} \mathrm{Li}\left({ }^{6} \mathrm{Li}, \alpha \alpha\right){ }^{4} \mathrm{He}$ & ${ }^{6} \mathrm{Li}$ & {$[23]$} \\
${ }^{6} \mathrm{Li}\left({ }^{3} \mathrm{He}, \alpha \alpha\right) \mathrm{H}$ & ${ }^{3} \mathrm{He}$ & {$[23]$} \\
${ }^{7} \mathrm{Li}(\mathrm{d}, \alpha \alpha) \mathrm{n}$ & $\mathrm{d}$ & {$[23,25]$} \\
${ }^{7} \mathrm{Li}\left({ }^{3} \mathrm{He}, \alpha \alpha\right){ }^{2} \mathrm{H}$ & ${ }^{3} \mathrm{He}$ & {$[23,25]$} \\
${ }^{2} \mathrm{H}\left({ }^{6} \mathrm{Li}, \mathrm{pt}\right){ }^{4} \mathrm{He}$ & ${ }^{6} \mathrm{Li}$ & {$[24]$} \\
${ }^{2} \mathrm{H}\left({ }^{3} \mathrm{He}, \mathrm{pt}\right) \mathrm{H}$ & ${ }^{3} \mathrm{He}$ & {$[26]$} \\
\hline
\end{tabular}

its applications: the target/projectile break-up invariance [21], the spectator invariance [22-24] and the possible use of virtual neutron beams [25, 27]. In recent works [22, 23] the spectator invariance was extensively examined for the ${ }^{6} \mathrm{Li}\left({ }^{6} \mathrm{Li}, \alpha \alpha\right){ }^{4} \mathrm{He}$ and the ${ }^{6} \mathrm{Li}\left({ }^{3} \mathrm{He}, \alpha \alpha\right) \mathrm{H}$ case as well as the ${ }^{7} \mathrm{Li}(\mathrm{d}, \alpha \alpha)$ n and ${ }^{7} \mathrm{Li}\left({ }^{3} \mathrm{He}, \alpha \alpha\right){ }^{2} \mathrm{H}$ reactions, thus comparing results arising from ${ }^{6} \mathrm{Li}$ and ${ }^{3} \mathrm{He}$ and deuteron and ${ }^{3} \mathrm{He}$ break-up respectively [23]. Agreement between the sets of data was found below and above the Coulomb barrier. The results already obtained are reported in table 1 with all the complete references. 


\section{Study of the $d(d, p) t$ reaction}

The idea of the present paper is to see whether the same can hold also for the $\mathrm{d}(\mathrm{d}, \mathrm{p}) \mathrm{t}$ binary reaction, studied via the quasi free ${ }^{2} \mathrm{H}\left({ }^{6} \mathrm{Li}, \mathrm{pt}\right){ }^{4} \mathrm{He}$ and ${ }^{2} \mathrm{H}\left({ }^{3} \mathrm{He}, \mathrm{pt}\right) \mathrm{H}$ reactions after ${ }^{6} \mathrm{Li}$ and ${ }^{3} \mathrm{He}$ break-up, respectively.

In Fig. 1, the different break-up schemes of interest are depicted. On the left side we report the QF break-up process which proceeds through ${ }^{6} \mathrm{Li}$, while on the right it is displayed the one which goes through ${ }^{3} \mathrm{He}$. The two

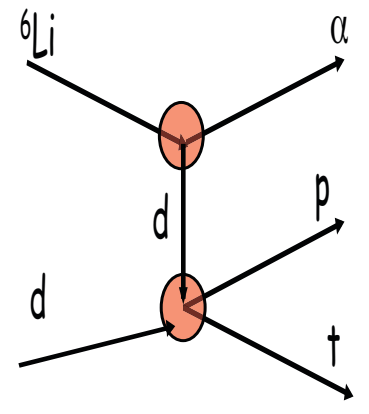

(a)

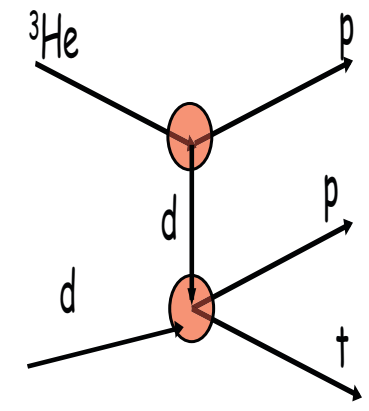

(b)
Figure 1. Sketch of the processes discussed in the text. Left (a): the quasi-free reaction involving the ${ }^{6} \mathrm{Li}$ break-up is shown. Right (b): the ${ }^{3} \mathrm{He}$ break-up is reported.

experiments are discussed extensively elsewhere, in particular the ${ }^{2} \mathrm{H}\left({ }^{6} \mathrm{Li}, \mathrm{pt}\right){ }^{4} \mathrm{He}$ reaction in $[24,29]$ and the ${ }^{2} \mathrm{H}\left({ }^{3} \mathrm{He}, \mathrm{pt}\right) \mathrm{H}$ in [34]. In both cases the standard prescriptions of the THM, as discussed in [20, 28], to extract the energy trend of the $\mathrm{S}(\mathrm{E})$-factor were applied. Therefore the binary cross section is extracted from the measured three-body one, in both experiments. The momentum distributions adopted for the data extractions were treated as prescribed in [31] and fitted with a Hänckel function for the ${ }^{6} \mathrm{Li}$ break-up (most suitable for the ${ }^{6} \mathrm{Li}$ case, as discussed in details in [30]) and with the Eckart function for the ${ }^{3} \mathrm{He}$ case.

The averaged results for the $\mathrm{d}(\mathrm{d}, \mathrm{p}) \mathrm{t}$ reaction after ${ }^{6} \mathrm{Li}$ break-up (red dots, see [24] for details) are then compared (see figure 2) with the ones extracted from ${ }^{3} \mathrm{He}$ break-up (see [34],). Data, expressed in the form of the astrophysical $S(E)$-factor for the binary $d(d, p) t$ reaction, were extracted from the measured three-body cross section according to the standard THM prescriptions [20] and in the framework of the Plane Wave Impulse Approximation. We can point out that the errors in the ${ }^{6} \mathrm{Li}$ break-up case are much larger than in the case of ${ }^{3} \mathrm{He}$ breakup. This is mainly due to the presence of the sequential mechanism in ${ }^{7} \mathrm{Li}$, already discussed in [29] that decreases the number of the QF events. Also the normalization errors and errors connected to the penetrability factor are fully included in

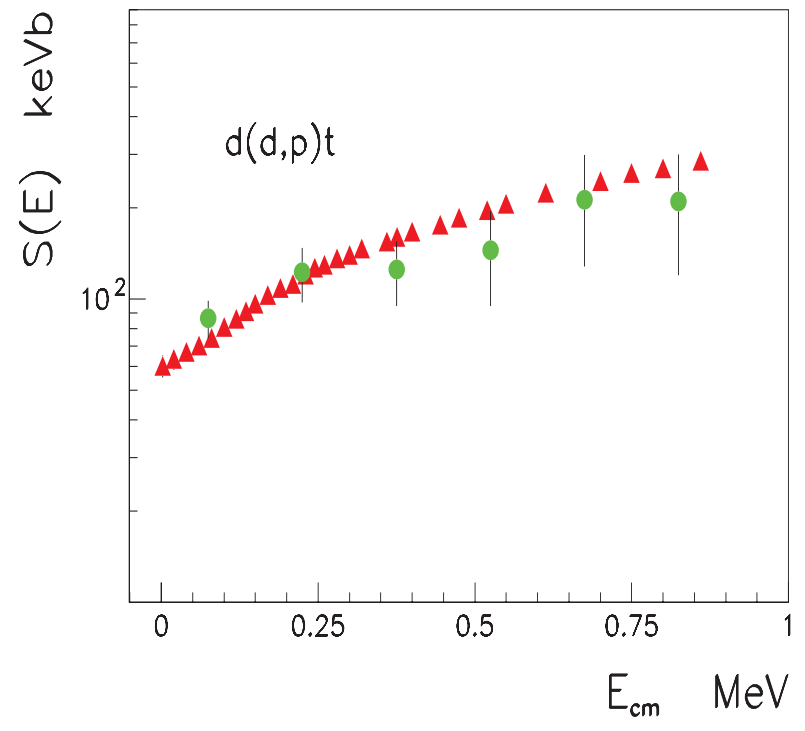

Figure 2. Averaged astrophysical $S(E)$-factor for the d(d,p)t reaction measured via THM after ${ }^{6} \mathrm{Li}$ break-up ( dots) and after ${ }^{3} \mathrm{He}$ break-up(triangles), extracted from [34] clearly showing the Trojan Horse particle invariance.

the error bars shown in the pictures. Polynomial fits were then performed on the data giving $\mathrm{S}_{0}=75 \pm 21 \mathrm{keV} \cdot \mathrm{b}$ in the case of the ${ }^{6} \mathrm{Li}$ break-up, while for ${ }^{3} \mathrm{He}$ one obtains $\mathrm{S}_{0}=58$ $\pm 2 \mathrm{keV} \cdot \mathrm{b}$. The results are in agreement, within the experimental errors, also with previous direct measurements [32, 33]. Coherent results from both the considered breakup schemes (as in figure 1) are achieved, not only in terms of the $\mathrm{S}(\mathrm{E})$-factors but also for the electron screening effect.

\section{Study of ${ }^{6} \mathrm{Li}(\mathrm{d}, \alpha)^{4} \mathrm{He}$}

The present experiment was aimed to study the ${ }^{6} \mathrm{Li}(\mathrm{d}, \alpha){ }^{4} \mathrm{He}$ reaction by means of the THM applied to the ${ }^{6} \mathrm{Li}\left({ }^{3} \mathrm{He}, \alpha \alpha\right) \mathrm{H}$ three-body reaction (see figure 3$)$. The measured cross section, extracted by the THM, is compared in the $E_{c m}=0.4-5 \mathrm{MeV}$ energy range with several data sets present extracted from ${ }^{6} \mathrm{Li}$ break-up (Figure 4). The agreement is very good throughout the whole energy range after a separate normalization of the indirect to direct data. Moreover the resonance at about $3 \mathrm{MeV}$ (corresponding to the $25.2 \mathrm{MeV}, 2^{+}$, energy level in ${ }^{8} \mathrm{Be}$ ) is clearly reproduced in the data set arising from ${ }^{3} \mathrm{He}$ breakup.

The investigation of this energy range is not relevant for astrophysical implications for the ${ }^{6} \mathrm{Li}$ depletion [6] but it provides a strong validity test for THM. In fact, as in the excitation function extracted in an indirect way does indeed reproduce the direct data both below and above the Coulomb barrier. Another interesting aspect of this analysis is the possibility to study the pole invariance of the quasi-free mechanism [22]. It is assumed, in fact, that changing the spectator particle in the quasi-free 
(a)
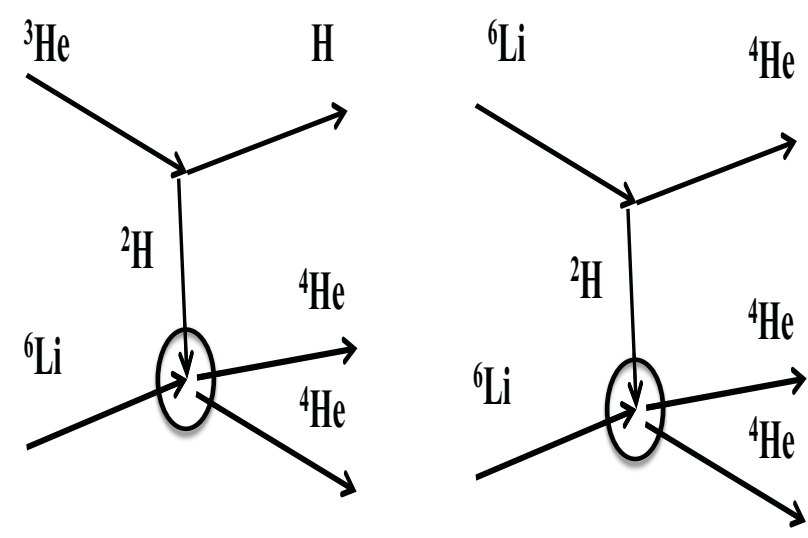

Figure 3. Sketch of the ${ }^{6} \mathrm{Li}(\mathrm{d}, \alpha)^{4} \mathrm{He}$ processes discussed in the text. Left (a): the quasi-free reaction involving the ${ }^{6} \mathrm{Li}$ break-up is shown. Right (b): the ${ }^{3} \mathrm{He}$ break-up is reported.

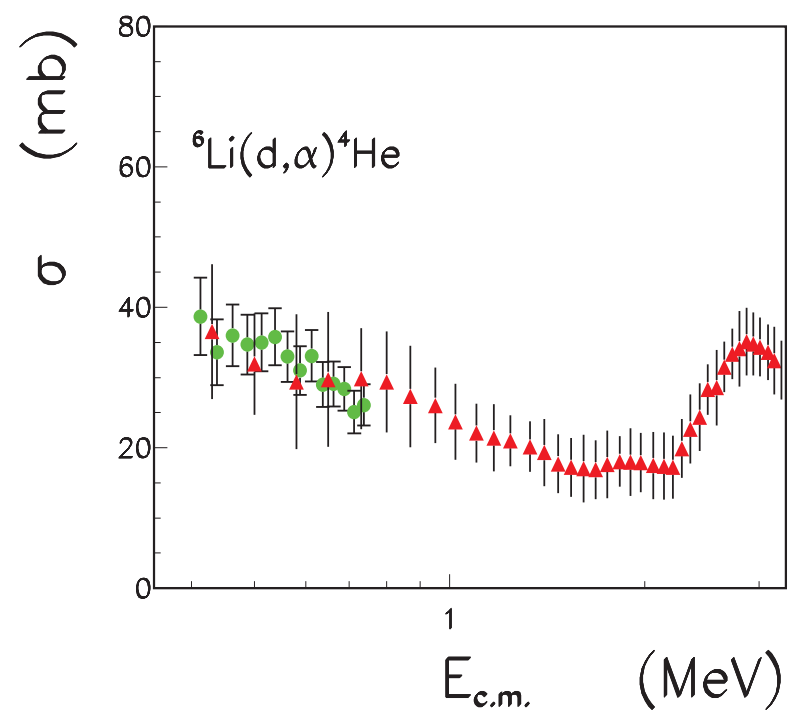

Figure 4. Excitation function for the ${ }^{6} \mathrm{Li}(\mathrm{d}, \alpha)^{4} \mathrm{He}$ reaction extracted by means of THM. The indirect data (circles) arising from ${ }^{6} \mathrm{Li}$ break-up are compared with those extracted from ${ }^{3} \mathrm{He}$ (triangles). The agreement is clearly within the experimental errors.

process (on which is founded the THM) does not give any change to the binary reaction of interest. This effect has been already explored in the case of the ${ }^{7} \mathrm{Li}(\mathrm{p}, \alpha)^{4} \mathrm{He}$ reaction [22]. In the case of the ${ }^{6} \mathrm{Li}(\mathrm{d}, \alpha)^{4} \mathrm{He}$ reaction we can see that in the energy range $0.4-1 \mathrm{MeV}$ we can compare data for the ${ }^{6} \mathrm{Li}(\mathrm{d}, \alpha)^{4} \mathrm{He}$ arising from the ${ }^{6} \mathrm{Li}\left({ }^{3} \mathrm{He}, \alpha \alpha\right) \mathrm{H}$ reaction (present work) with the ones extracted from ${ }^{6} \mathrm{Li}\left({ }^{6} \mathrm{Li}, \alpha \alpha\right){ }^{4} \mathrm{He}[4,21]$ (see Fig. 4). The agreement is very good within the experimental errors. This strengthens a lot the present knowledge of the Trojan Horse Method and makes the application of the method more straightforward even with a simplified approach.

This work was supported in part by the Italian Ministry of the University under Grant No. RBFR082838 and by the Italian Ministry of University MIUR under the grant "LNS Astrofisica Nucleare (fondi premiali)". A.M. M. acknowledges the support of the US Department of Energy under Grant Nos. DE-FG02-93ER40773, DE-FG5209NA29467, and DE-SC0004958 (topical collaboration TORUS). C.B. acknowledges the support of the US Department of Energy under Grant Nos. DE-SC0004971, DE-FG02-08ER41533.

\section{References}

[1] R. E. Tribble, C. A. Bertulani et al., Rep. Prog. Phys. 77, 116901 (2014)

[2] A. Mukhamedzhanov, P. Bem et al., Phys. Rev. C 78, 015804 (2008)

[3] M. Lattuada, R. G. Pizzone et al., Ap. J. 562, 1076 (2001)

[4] C. Spitaleri, S. Typel et al., Phys. Rev. C 63, 055801(2001)

[5] A. Tumino, C. Spitaleri et al., Phys. Rev. C 78 , 064001 (2008)

[6] R. G. Pizzone, C. Spitaleri et al., A. \& A. 398, 423 (2003)

[7] R. G. Pizzone, C. Spitaleri et al., A. \& A. 438, 779 (2005)

[8] S. Palmerini ,M. L. Sergi et al., ApJ 764, 128 (2013)

[9] L. Lamia et al., Nucl. Phys. A A 787 , 309C-314C (2007)

[10] L. Lamia, C. Spitaleri et al., Jour. Phys. G, 39, 015106 (2012)

[11] R. G. Pizzone, C. Spitaleri et al., ApJ 786, 112 (2014)

[12] L. Lamia, C. Spitaleri et al., Phys. Rev. C 85, 025805 (2012)

[13] L. Lamia, C. Spitaleri et al., A. \& A. 541, 158 (2012)

[14] L. Lamia, C. Spitaleri et al., 2013, Ap. J. 768, 65

[15] S. Romano et al., Eur. Phys. J. A, 27, 221 (2006)

[16] Q. Wen, C. Li et al., Phys. Rev. C 78, 035805 (2008)

[17] L. Lamia, C. Spitaleri et al., Nuovo Cimento C 31, 423 (2008)

[18] M. Gulino, C. Spitaleri et al., Phys. Rev. C 87, 012801(R) (2013)

[19] C. Spitaleri, S. Cherubini et al., Nucl. Phys. A, 719, 99c (2003).

[20] C. Spitaleri, A. Mukhamedzhanov et al., Physics of At. Nucleus, 74, 1725 (2011)

[21] A. Musumarra, R. G. Pizzone et al., Phys. Rev. C 64, 068801 (2001)

[22] A. Tumino et al., Eur. Phys. J. A 27 Supplement 1, 243 (2006)

[23] R. G.Pizzone, C. Spitaleri et al., Phys. Rev. C, 83, 045801 (2011)

[24] R. G.Pizzone, C. Spitaleri et al., Phys. Rev. C, 87, 025805 (2013) 
[25] A. Tumino, C. Spitaleri et al., Eur. Phys. J. A 25, 649 (2005)

[26] A. Tumino, C. Spitaleri et al., ApJ 785, 96 (2014)

[27] M. Gulino, C. Spitaleri et al., Jour. of Phys. 37 , 125105 (2010)

[28] C. Spitaleri, L Lamia et al., Phys. Rev. C 90, 035801 (2014)

[29] A. Rinollo et al., Nucl. Phys. A, 758, 146c (2003)
[30] R. G. Pizzone, C. Spitaleri et al., Phys. Rev. C 71, 058801 (2005)

[31] R. G. Pizzone, C. Spitaleri et al., Phys. Rev. C 80, 025807 (2009)

[32] A. Krauss, C. Rolfs et al., Nucl. Phys. A, 465 (1987)

[33] R. E. Brown \& N. Jarmie, Phys. Rev. C, 41, 1391 (1990)

[34] A. Tumino, C. Spitaleri et al., Phys. Lett. B, 705, 546 (2011) 